Faculty of Mathematical Sciences

\section{University of Twente}

University for Technical and Social Sciences
P.O. Box 217

7500 AE Enschede

The Netherlands

Phone: +31-53-4893400

Fax: +31-53-4893114

Email: memo@math.utwente.nl

Memorandum No. 1561

Consistency and potentials

in cooperative TU-games:

Sobolev's reduced game revived

T.S.H. DRIESSEN

DeCember 2000

ISSN 0169-2690 


\title{
Consistency and Potentials in Cooperative TU-Games: Sobolev's Reduced Game Revived
}

\author{
Theo DRIESSEN *
}

\begin{abstract}
It was a quarter of a century ago that Sobolev proved the reduced game (otherwise called consistency) property for the much-discussed Shapley value of cooperative TUgames. The purpose of this paper is to extend Sobolev's result in two ways. On the one hand the unified approach applies to the enlarged class consisting of game-theoretic solutions that possess a so-called potential representation; on the other Sobolev's reduced game is strongly adapted in order to establish the consistency property for solutions that admit a potential. Actually, Sobolev's explicit description of the reduced game is now replaced by a similar, but implicit definition of the modified reduced game; the characteristic function of which is implicitly determined by a bijective mapping on the universal game space (induced by the solution in question). The resulting consistency property solves an outstanding open problem for a wide class of game-theoretic solutions. As usual, the consistency together with some kind of standardness for two-person games fully characterize the solution. A detailed exposition of the developed theory is given in the event of dealing with so-called semivalues of cooperative TU-games and the Shapley and Banzhaf values in particular.
\end{abstract}

Keywords: cooperative TU-game, reduced game, solution, consistency, potential, semivalue

1991 Mathematics Subject Classifications: Primary 90D12, Secondary 90D40

\section{Introduction}

In physics a vector field $u$ is said to be conservative if there exists a continuously differentiable function $U$ called potential the gradient of which agrees with the vector field (notation: $\nabla U=$ $u$ ). There exist several characterizations of conservative vector fields (e.g., $\nabla_{j} u_{i}=\nabla_{i} u_{j}$, or every contour integral with respect to the vector field $u$ is zero). Surprisingly, the successful treatment of the potential in physics turned out to be reproducible, in the late eighties, in the mathematical field called cooperative game theory. Informally, a solution concept $\psi$ on the universal game space $\mathcal{G}$ is said to possess a potential representation if it is the discrete gradient of a real-valued function $P$ on $\mathcal{G}$ called potential (notation: $\nabla P=\psi$ ). In other words, if possible, each component of the game-theoretic solution may be interpreted as the incremental return with respect to the potential function. In their innovative paper, Hart and Mas-Colell (cf. [6]) showed that the well-known game-theoretic solution called Shapley

${ }^{*}$ Theo S.H. Driessen, Faculty of Mathematical Sciences, University of Twente, P.O. Box 217, 7500 AE Enschede, The Netherlands. E-mail: t.s.h.driessen@math.utwente.nl 
value is the unique solution that has a potential representation and meets the efficiency principle as well. In the second stage (the nineties) of the potential research into the solution part of cooperative game theory, various researchers contributed different, but equivalent characterizations of (not necessarily efficient) solutions that admit a potential (cf. [9], [1], [11]). Almost all of these characterizations of solutions, stated in terms of the potential approach applied in cooperative game theory, resemble similar ones stated in physical terminology. For instance, the characterization $\nabla_{j} u_{i}=\nabla_{i} u_{j}$ of a conservative vector field $u$ is analogous to its discrete version $\nabla_{j} \psi_{i}=\nabla_{i} \psi_{j}$ with respect to a game-theoretic solution $\psi$, commonly known as the law of preservation of discrete differences (cf. [9]), otherwise called the balanced contributions principle (cf. [1], [8], [11]).

One characterization with no counterpart in physics states that a game-theoretic solution possesses a potential representation if and only if the solution for any game equals the Shapley value of another game induced by both the initial game and the relevant solution concept (cf. [1]). Our main goal is to exploit this particular characterization whenever one deals with the so-called reduced game (or consistency) property for solutions. Generally speaking, the consistency property is a very powerful and widely used tool to axiomatize game-theoretic solutions (cf. the surveys on consistency in [4], [7]). In the early seventies Sobolev (cf. [13]) established the consistency property for the well-known Shapley value with respect to an appropriately chosen reduced game. With Sobolev's result at hand, we are in a position to establish, under certain circumstances, the consistency property for a solution that has a potential representation. For that purpose the consistency property is formulated with respect to a strongly adapted version of the reduced game used by Sobolev. Section 2 is devoted to the whole treatment of the relevant consistency property (see Theorem 2.4) the proof of which is based on the particular characterization of solutions that admit a potential. The resulting consistency property solves an outstanding open problem for a wide class of solutions. Moreover, we add an axiomatization for these solutions in terms of their consistency property, together with some kind of standardness for two-person games (see Theorem 2.6). Our modified reduced game differs from Sobolev's reduced game only in that any game is replaced by its image under a bijective mapping on the universal game space (induced by the solution in question). The particular bijective mapping, induced by the Shapley value, equals the identity. To be exact, Sobolev's explicit description of the reduced game refers to the initial game itself, whereas our similar, but implicit definition of the modified reduced game is formulated in terms of the image of both the modified reduced game and the initial game (see Theorem 2.4).

In the general framework concerning an arbitrary solution that admits a potential, there is no way to acquire more information about the associated bijective mapping and consequently, the implicit definition of the modified reduced game can not be explored any further to strengthen the consistency property for this solution. For a certain type of solutions called semivalues (cf. [5]), however, the associated bijective mapping and its inverse are computable and hence, under these particular circumstances, one gains an insight into the modified reduced game itself. Section 3 is devoted to the whole treatment of these semivalues and, in the setting of the consistency property for these semivalues, we provide various elegant interpretations of the modified reduced game (see Theorems 3.3 and 3.4). 


\section{Consistency property for solutions that admit a potential}

A cooperative game with transferable utility (TU) is a pair $\langle N, v\rangle$, where $N$ is a nonempty, finite set and $v: 2^{N} \rightarrow \mathbb{R}$ is a characteristic function, defined on the power set of $N$, satisfying $v(\emptyset):=0$. An element of $N$ (notation: $i \in N$ ) and a nonempty subset $S$ of $N$ (notation: $S \subseteq N$ or $S \in 2^{N}$ with $S \neq \emptyset$ ) is called a player and coalition respectively, and the associated real number $v(S)$ is called the worth of coalition $S$. The size (cardinality) of coalition $S$ is denoted by $|S|$ or, if no ambiguity is possible, by $s$. Particularly, $n$ denotes the size of the player set $N$. Given a (transferable utility) game $\langle N, v\rangle$ and a coalition $S$, we write $\langle S, v\rangle$ for the subgame obtained by restricting $v$ to subsets of $S$ only (i.e., to $2^{S}$ ). Let $\mathcal{G}$ denote the set of all cooperative games with an arbitrary player set, whereas $\mathcal{G}^{N}$ denotes the (vector) space of all games with reference to a player set $N$ which is fixed beforehand.

Concerning the solution theory for cooperative TU-games, the paper is devoted to singlevalued solution concepts. Formally, a solution $\psi$ on $\mathcal{G}$ (or on a particular subclass of $\mathcal{G}$ ) associates a single payoff vector $\psi(N, v)=\left(\psi_{i}(N, v)\right)_{i \in N} \in \mathbb{R}^{N}$ with every TU-game $\langle N, v\rangle$. The so-called value $\psi_{i}(N, v)$ of player $i$ in the game $\langle N, v\rangle$ represents an assessment by $i$ of his gains from participating in the game. Until further notice, no constraints are imposed upon a solution $\psi$ on $\mathcal{G}$. In the next definition we present two key notions (out of four).

Definition 2.1. (cf. [1], [2], [6], [9], [11]) Let $\psi$ be a solution on $\mathcal{G}$.

(i) We say the solution $\psi$ admits a potential if there exists a function $P_{\psi}: \mathcal{G} \rightarrow \mathbb{R}$ satisfying

$$
P_{\psi}(N, v)-P_{\psi}(N \backslash\{i\}, v)=\psi_{i}(N, v) \quad \text { for all }\langle N, v\rangle \in \mathcal{G} \text { and all } i \in N .
$$

(ii) The mapping $F_{\psi}: \mathcal{G} \rightarrow \mathcal{G}$ associates with any game $\langle N, v\rangle$ its solution game $\left\langle N, F_{\psi}^{v}\right\rangle$; the characteristic function of which is defined to be

$$
F_{\psi}^{v}(S):=\sum_{i \in S} \psi_{i}(S, v) \quad \text { for all } S \subseteq N, S \neq \emptyset
$$

In words, the potential function $P_{\psi}$ represents a scalar evaluation for cooperative TU-games, of which any player's marginal contribution agrees with the player's value according to the relevant solution $\psi$ (notation: $\nabla P_{\psi}=\psi$ ). If the potential exists, it is uniquely determined up to an additive constant by the recursive formula $n \cdot P_{\psi}(N, v)-\sum_{i \in N} P_{\psi}(N \backslash\{i\}, v)=F_{\psi}^{v}(N)$. Usually, it is tacitly assumed that the potential is zero-normalized (i.e., $P_{\psi}(\emptyset, v):=0$ ). In fact, it is well-known that the potential function $P_{\psi}$ (if it exists) is given by

$$
P_{\psi}(N, v)=\sum_{\substack{S \subseteq N \\
S \neq \emptyset}} \frac{F_{\psi}^{v}(S)}{s \cdot\left(\begin{array}{l}
n \\
s
\end{array}\right)} \quad \text { for all }\langle N, v\rangle \in \mathcal{G} .
$$

By (2.2), the worth $F_{\psi}^{v}(S)$ of coalition $S$ in the solution game $\left\langle N, F_{\psi}^{v}\right\rangle$ represents the overall gains (according to the solution $\psi$ ) to the members of $S$ from participating in the induced subgame $\langle S, v\rangle$ (on the understanding that players outside $S$ are not supposed to cooperate). Generally speaking, the solution game differs from the initial game. Notice that both games are the same if and only if the solution $\psi$ meets the efficiency principle, i.e.,

$$
\sum_{i \in N} \psi_{i}(N, v)=v(N) \quad \text { for all }\langle N, v\rangle \in \mathcal{G} .
$$


The core topic involves the so-called consistency treatment for solutions that admit a potential. For that purpose, we need to recall one basic theorem from Calvo's paper [1]; the main result of which is referring to the well-known Shapley value. With the help of Sobolev's pioneer work [13] in the early seventies on the consistency property for the Shapley value, we are able to prove, under certain circumstances, a similar consistency property for (not necessarily efficient) solutions that admit a potential.

Theorem 2.2. Consider the setting of Definition 2.1.

(i) (cf. [1], Theorem, page 178) Let $\psi$ be a solution on $\mathcal{G}$. Then $\psi$ admits a potential if and only if $\psi(N, v)=\operatorname{Sh}\left(N, F_{\psi}^{v}\right)$ for all $\langle N, v\rangle \in \mathcal{G}$. That is, any solution that admits a potential equals the Shapley value of the associated solution game.

(ii) (cf. [6], Theorem A, page 591) The Shapley value is the unique solution $\psi$ on $\mathcal{G}$ that admits a potential and is efficient as well. Here the Shapley value $S h_{i}(N, v)$ of player $i \in N$ in the $n$-person game $\langle N, v\rangle$ is defined as follows (cf. [12]):

$$
S h_{i}(N, v)=\sum_{S \subseteq N \backslash\{i\}} \frac{1}{n \cdot\left(\begin{array}{c}
n-1 \\
s
\end{array}\right)} \cdot[v(S \cup\{i\})-v(S)] \quad \text { for all }\langle N, v\rangle \in \mathcal{G} \text {, all } i \in N \text {. }
$$

Theorem 2.3. (cf. [13], [4])

With an $n$-person game $\langle N, v\rangle$, a player $i \in N$, and his payoff $x_{i} \in \mathbb{R}$ (provided $n \geq 2$ ), there is associated the reduced game $\left\langle N \backslash\{i\}, v_{N \backslash\{i\}}^{x_{i}}\right\rangle$ with player set $N \backslash\{i\}$ defined to be

$$
v_{N \backslash\{i\}}^{x_{i}}(S):=\frac{s}{n-1} \cdot\left[v(S \cup\{i\})-x_{i}\right]+\frac{n-1-s}{n-1} \cdot v(S) \quad \text { for all } S \subseteq N \backslash\{i\} .
$$

Then the Shapley value $S h$ on $\mathcal{G}$ is consistent with respect to this reduced game, i.e.,

$$
S h_{j}\left(N \backslash\{i\}, v_{N \backslash\{i\}}^{S h_{i}(N, v)}\right)=S h_{j}(N, v) \quad \text { for all }\langle N, v\rangle \in \mathcal{G} \text {, all } i \in N \text {, all } j \in N \backslash\{i\} .
$$

That is, there is no inconsistency in what each of the players in the reduced game will get according to the Shapley value, in either the reduced game or the initial game.

Now we are in a position to state and prove a similar consistency property for solutions that admit a potential. Actually, for a given solution $\psi$, the appropriately chosen reduced game resembles Sobolev's reduced game (2.4), but they differ in that the initial game $v$ is replaced by the associated solution game $F_{\psi}^{v}$. In summary, it turns out that the cornerstone of the consistency approach to (not necessarily efficient) solutions is the solution game instead of the game itself. Consequently, we have to define the modified reduced game implicitly by means of its associated solution game, on the understanding that a one-to-one correspondence (bijection) between games and solution games is supposed to be available. ${ }^{1}$

\footnotetext{
${ }^{1}$ In [2] (Definition 11, page 459), the solution game plays an identically prominent role in defining the reduced game; the characteristic function of which is, however, from a different type since it deals with the reduced game in the sense of Hart and Mas-Colell (cf. [6]). Our model deals with the reduced game in the sense of Sobolev.
} 
Theorem 2.4. Let $\psi$ be a solution on $\mathcal{G}$ that admits a potential. Suppose that the induced mapping $F_{\psi}: \mathcal{G} \rightarrow \mathcal{G}$, as given by $(2.2)$, is a bijection.

With an $n$-person game $\langle N, v\rangle$, a player $i \in N$, and his payoff $y_{i} \in \mathbb{R}$ (provided $n \geq 2$ ), there is associated the modified reduced game $\left\langle N \backslash\{i\}, \tilde{v}_{N \backslash\{i\}}^{y_{i}}\right\rangle$ with player set $N \backslash\{i\}$ which is defined implicitly by its associated solution game $\left\langle N \backslash\{i\}, F_{\psi}^{\left(\tilde{v}_{N i}^{y_{i}} \backslash i\right\}}\right\rangle$; the characteristic function of which is defined to be

$F_{\psi}^{\left(\tilde{v}_{N \backslash\{i\}}^{y_{i}}\right)}(S):=\frac{s}{n-1} \cdot\left[F_{\psi}^{v}(S \cup\{i\})-y_{i}\right]+\frac{n-1-s}{n-1} \cdot F_{\psi}^{v}(S) \quad$ for all $S \subseteq N \backslash\{i\}$.

Then the solution $\psi$ on $\mathcal{G}$ is consistent with respect to this modified reduced game, i.e.,

$$
\psi_{j}\left(N \backslash\{i\}, \tilde{v}_{N \backslash\{i\}}^{\psi_{i}(N, v)}\right)=\psi_{j}(N, v) \quad \text { for all }\langle N, v\rangle \in \mathcal{G} \text {, all } i \in N \text {, all } j \in N \backslash\{i\} .
$$

That is, there is no inconsistency in what each of the players in the reduced game will get according to the solution $\psi$, in either the reduced game or the initial game.

\section{Proof of Theorem 2.4.}

Fix both the $n$-person game $\langle N, v\rangle$ and a player $i \in N$ (where $n \geq 2$ ). Write $w$ instead of $F_{\psi}^{v}$. Since $\psi$ admits a potential, it holds, by Theorem 2.2(i), $\psi(N, v)=\operatorname{Sh}\left(N, F_{\psi}^{v}\right)=\operatorname{Sh}(N, w)$. The essential part of the proof concerns the claim that the solution game (2.6) technique applied to the modified reduced game agrees with Sobolev's reduced game (2.4) technique applied to the initial solution game. Formally, we claim the following:

$$
\left\langle N \backslash\{i\}, F_{\psi}^{\left(\tilde{v}_{N \backslash\{i\}}^{\psi_{i}(N, v)}\right)}\right\rangle=\left\langle N \backslash\{i\}, w_{N \backslash\{i\}}^{S h_{i}(N, w)}\right\rangle
$$

Indeed, from both types of reduced games, we deduce that, for all $S \subseteq N \backslash\{i\}$, it holds

$$
\begin{aligned}
F_{\psi}^{\left(\tilde{v}_{N \backslash\{i\}}^{\psi_{i}(N, v)}\right)}(S) & \stackrel{(2.6)}{=} \frac{s}{n-1} \cdot\left[F_{\psi}^{v}(S \cup\{i\})-\psi_{i}(N, v)\right]+\frac{n-1-s}{n-1} \cdot F_{\psi}^{v}(S) \\
& =\frac{s}{n-1} \cdot\left[w(S \cup\{i\})-S h_{i}(N, w)\right]+\frac{n-1-s}{n-1} \cdot w(S) \\
& \stackrel{(2.4)}{=} w_{N \backslash\{i\}}^{S h_{i}(N, w)}(S)
\end{aligned}
$$

This proves (2.8). From this we deduce that the following chain of four equalities holds:

$$
\begin{aligned}
\psi_{j}\left(N \backslash\{i\}, \tilde{v}_{N \backslash\{i\}}^{\psi_{i}(N, v)}\right) & =\quad S h_{j}\left(N \backslash\{i\}, F_{\psi}^{\left(\tilde{v}_{N \backslash\{i\}}^{\psi_{i}(N, v)}\right)}\right) \\
& \stackrel{(2.8)}{=} S h_{j}\left(N \backslash\{i\}, w_{N \backslash\{i\}}^{S h_{i}(N, w)}\right) \\
& \stackrel{(2.5)}{=} \quad S h_{j}(N, w) \\
= & \psi_{j}(N, v) \quad \text { for all } j \in N \backslash\{i\} .
\end{aligned}
$$

where the first and last equality are due to Theorem 2.2(i) and the third equality is due to Theorem 2.3 concerning the consistency property (2.5) for the Shapley value. This completes the full proof of the consistency property for the solution $\psi$. 
Definition 2.5. Let $\psi$ and $\Phi$ be two solutions on $\mathcal{G}$. We say the solution $\Phi$ is $F_{\psi}$-standard for two-person games if, for every two-person game $\langle\{i, j\}, v\rangle$ and every player $k \in\{i, j\}$, it holds that

$$
\Phi_{k}(\{i, j\}, v)=F_{\psi}^{v}(\{k\})+\frac{1}{2} \cdot\left[F_{\psi}^{v}(\{i, j\})-F_{\psi}^{v}(\{i\})-F_{\psi}^{v}(\{j\})\right]
$$

Clearly, by (2.1)-(2.2), a solution $\psi$ that admits a (zero-normalized) potential, verifies the $F_{\psi^{-}}$ standardness for two-person games. We conclude this section with the next axiomatization.

Theorem 2.6. Let $\psi$ be a solution on $\mathcal{G}$ that admits a potential. Suppose that the induced mapping $F_{\psi}: \mathcal{G} \rightarrow \mathcal{G}$, as given by $(2.2)$, is a bijection. Then $\psi$ is the unique solution $\Phi$ on $\mathcal{G}$ that verifies the following two properties:

(i) Consistency with respect to the modified reduced game implicitly defined through its associated solution game (2.6) (with reference to the given solution $\psi$ ).

(ii) $F_{\psi}$-standardness for two-person games.

\section{Proof of the uniqueness part of Theorem 2.6.}

Besides the given solution $\psi$, suppose that a solution $\Phi$ on $\mathcal{G}$ verifies the consistency property and the $F_{\psi}$-standardness for two-person games. We prove by induction on the size $n$ of the player set $N$ that $\Phi(N, v)=\psi(N, v)$ for every game $\langle N, v\rangle$. The case $n=2$ holds trivially because of the $F_{\psi}$-standardness for two-person games applied to both solutions. From now on fix an $n$-person game $\langle N, v\rangle$ with $n \geq 3$. Due to the induction hypothesis, it holds that $\Phi(M, u)=\psi(M, u)$ for every game $\langle M, u\rangle$ with $2 \leq|M| \leq n-1$. Note that, for all $i \in N$, all $x_{i} \in \mathbb{R}, y_{i} \in \mathbb{R}$, it follows immediately from (2.6) that

$$
F_{\psi}^{\left(\tilde{v}_{N \backslash\{i\}}^{y_{i}}\right)}(S)=F_{\psi}^{\left(\tilde{v}_{N \backslash\{i\}}^{x_{i}}\right)}(S)+s \cdot \frac{x_{i}-y_{i}}{n-1} \quad \text { for all } S \subseteq N \backslash\{i\} .
$$

In other words, the two solution games $\left\langle N \backslash\{i\}, F_{\psi}^{\left(\tilde{v}_{N \backslash\{i\}}^{y_{i}}\right)}\right\rangle$ and $\left\langle N \backslash\{i\}, F_{\psi}^{\left(\tilde{v}_{N \backslash\{i\}}^{x_{i}}\right)}\right\rangle$ are strategically equivalent (with reference to the translation vector $\frac{x_{i}-y_{i}}{n-1} \cdot(1,1, \ldots, 1) \in \mathbb{R}^{N \backslash\{i\}}$ ) and thus, the covariance property for the Shapley value $S h$ applies in the sense that it holds

$$
S h_{j}\left(N \backslash\{i\}, F_{\psi}^{\left(\tilde{v}_{N \backslash\{i\}}^{y_{i}}\right)}\right)=S h_{j}\left(N \backslash\{i\}, F_{\psi}^{\left(\tilde{v}_{N \backslash\{i\}}^{x_{i}}\right)}\right)+\frac{x_{i}-y_{i}}{n-1} \quad \text { for all } j \in N \backslash\{i\} .
$$

For all $i \in N$ and all $j \in N \backslash\{i\}$, we obtain the following chain of equalities:

$$
\begin{aligned}
& \Phi_{j}(N, v)=\Phi_{j}\left(N \backslash\{i\}, \tilde{v}_{N \backslash\{i\}}^{\Phi_{i}(N, v)} \quad \text { by consistency for } \Phi\right. \\
& =\psi_{j}\left(N \backslash\{i\}, \tilde{v}_{N \backslash\{i\}}^{\Phi_{i}(N, v)}\right) \quad \text { by induction hypothesis } \\
& =S h_{j}\left(N \backslash\{i\}, F_{\psi}^{\left(\tilde{v}_{N \backslash\{i\}}^{\Phi_{i}(N, v)}\right)}\right) \quad \text { by Theorem 2.2(i) } \\
& \stackrel{(2.9)}{=} S h_{j}\left(N \backslash\{i\}, F_{\psi}^{\left(\tilde{v}_{N \backslash i\}}^{\psi_{i}(N, v)}\right)}\right)+\frac{\psi_{i}(N, v)-\Phi_{i}(N, v)}{n-1} \quad \text { by covariance for } S h \\
& =\psi_{j}\left(N \backslash\{i\}, \tilde{v}_{N \backslash\{i\}}^{\psi_{i}(N, v)}\right)+\frac{\psi_{i}(N, v)-\Phi_{i}(N, v)}{n-1} \quad \text { by Theorem 2.2(i) } \\
& =\psi_{j}(N, v)+\frac{\psi_{i}(N, v)-\Phi_{i}(N, v)}{n-1} \quad \text { by consistency for } \psi
\end{aligned}
$$


We conclude that

$$
\Phi_{j}(N, v)-\psi_{j}(N, v)=\frac{\psi_{i}(N, v)-\Phi_{i}(N, v)}{n-1} \quad \text { for all } i \in N \text { and all } j \in N \backslash\{i\} .
$$

By interchanging the roles of players $i$ and $j$, the latter result yields

$$
\Phi_{i}(N, v)-\psi_{i}(N, v)=\frac{\Phi_{i}(N, v)-\psi_{i}(N, v)}{(n-1)^{2}} \quad \text { for all } i \in N .
$$

Since $n \geq 3$, we arrive at the conclusion that $\Phi_{i}(N, v)-\psi_{i}(N, v)=0$ for all $i \in N$. Thus, $\Phi(N, v)=\psi(N, v)$ for every game $\langle N, v\rangle$ as was to be shown.

\section{Consistency property for pseudovalues: a detailed exposi- tion}

In this section we aim to clarify that, if we deal with a particular type of solutions called pseudovalues, then various elegant interpretations arise in the study of the modified reduced game as given by (2.6). Besides the various appealing interpretations in some kind of terminology, we claim that the implicit definition of the modified reduced game can be transformed into an explicit one, although the resulting explicit description becomes rather laborious. In [5] a semivalue on $\mathcal{G}^{N}$ is defined to be a function $\psi: \mathcal{G}^{N} \rightarrow \mathbb{R}^{N}$ which verifies the linearity, symmetry, monotonicity, and projection axioms. It was shown in [5] (Theorem 1, page 123) that every semivalue is of the following form, the formula of which will be used as our starting point (but we omit certain non-negativity constraints). Throughout this section, lower-case letters $s, t, n$, and so on, are supposed to be non-negative integers because they are meant to refer to sizes of coalitions. For notation' sake, let $\mathcal{P}=\left\{p_{s}^{n}\right\}$ represent an arbitrary collection of real numbers called weights and meant to be read as $\mathcal{P}:=\left\{p_{s}^{n} \in \mathbb{R} \mid n \in\{1,2, \ldots\}, s \in\{0,1, \ldots, n-1\}\right\}$.

Definition 3.1. We say a solution $\psi$ on $\mathcal{G}$ is a pseudovalue on $\mathcal{G}$ if there exists a collection of weights $\mathcal{P}=\left\{p_{s}^{n}\right\}$ such that the following two conditions hold:

$$
\psi_{i}(N, v)=\sum_{S \subseteq N \backslash\{i\}} p_{s}^{n} \cdot[v(S \cup\{i\})-v(S)] \quad \text { for all }\langle N, v\rangle \in \mathcal{G} \text {, all } i \in N ;
$$

(ii) the collection of weights $\mathcal{P}=\left\{p_{s}^{n}\right\}$ possesses the upwards triangle property, i.e.,

$$
p_{s}^{n+1}+p_{s+1}^{n+1}=p_{s}^{n} \quad \text { for all } n \geq 1 \text { and all } 0 \leq s \leq n-1 .
$$

In words, in the setting of populations with a variable size, the "weight" of the formation of a coalition of size $s$ in an $n$-person population equals the sum of the "weights" of the two events which may arise by enlarging the population with one person (namely, two coalitions of consecutive sizes $s$ and $s+1$ respectively in an $(n+1)$-person population).

For reasons that will be explained later on, no further constraints are imposed upon the weights (e.g., they are not necessarily non-negative). A pseudovalue with reference to nonnegative weights is known as a semivalue (cf. [5]). It is straightforward to check that any 
pseudovalue $\psi$ admits a potential (due to the upwards triangle property for $\mathcal{P}=\left\{p_{s}^{n}\right\}$ ), where the potential function $P_{\psi}: \mathcal{G} \rightarrow \mathbb{R}$ is given by

$$
P_{\psi}(N, v)=\sum_{\substack{S \subseteq N, S \neq \emptyset}} p_{s-1}^{n} \cdot v(S) \quad \text { for all }\langle N, v\rangle \in \mathcal{G} .
$$

To start with, we determine an explicit formula for the associated solution game. As an adjunct, we become engaged with induced collections of weights verifying the upwards triangle property.

Proposition 3.2. Let $\psi$ be a pseudovalue on $\mathcal{G}$ of the form (3.1) associated with the collection of weights $\mathcal{P}=\left\{p_{s}^{n}\right\}$. Let $F_{\psi}: \mathcal{G} \rightarrow \mathcal{G}$ be the induced mapping as given by (2.2). Then the following holds:

$$
F_{\psi}^{v}(T)=\sum_{S \subseteq T}\left[s \cdot p_{s-1}^{t}-(t-s) \cdot p_{s}^{t}\right] \cdot v(S) \quad \text { for all }\langle N, v\rangle \in \mathcal{G}, \text { all } T \subseteq N .
$$

(ii) If the collection $\mathcal{P}=\left\{p_{s}^{n}\right\}$ possesses the upwards triangle property, so does the induced collection $\tilde{\mathcal{P}}=\left\{\tilde{p}_{s}^{n}\right\}$ defined by

$$
\tilde{p}_{s}^{n}:=(s+1) \cdot p_{s}^{n}-(n-1-s) \cdot p_{s+1}^{n} \quad \text { for all } n \geq 1 \text { and all } 0 \leq s \leq n-1 .
$$

(iii) For every $n \geq 1$, given that $\tilde{p}_{s}^{n}=(s+1) \cdot p_{s}^{n}-(n-1-s) \cdot p_{s+1}^{n}$ for all $0 \leq s \leq n-1$, then the weights $p_{s}^{n}$ can be re-discovered as follows:

$$
p_{s}^{n}=\frac{1}{n \cdot\left(\begin{array}{c}
n-1 \\
s
\end{array}\right)} \cdot \sum_{t=s}^{n-1}\left(\begin{array}{c}
n \\
t+1
\end{array}\right) \cdot \tilde{p}_{t}^{n} \quad \text { for all } 0 \leq s \leq n-1 .
$$

(iv) Suppose (3.5) holds for all $n \geq 1$. If the collection $\tilde{\mathcal{P}}=\left\{\tilde{p}_{s}^{n}\right\}$ possesses the upwards triangle property, so does the induced collection $\mathcal{P}=\left\{p_{s}^{n}\right\}$.

(v) The special case $p_{s}^{n}=\frac{1}{n \cdot\left(\begin{array}{c}n-1 \\ s\end{array}\right)}$ yields $\tilde{p}_{n-1}^{n}=1$ and $\tilde{p}_{s}^{n}=0$ for all $n \geq 1$, all $0 \leq s \leq n-2$.

For expositional convenience, the computational, but straightforward proof of Proposition 3.2 is postponed till the appendix. By (3.4)-(3.5), there exists a natural one-to-one correspondence between collections of weights that satisfy the upwards triangle property. Particularly, any pseudovalue $\psi$ induces another pseudovalue $\tilde{\psi}$, the weights of which are given by (3.4) (and vice versa, by (3.5)). For instance, by part (v), the Shapley value induces the pseudovalue $\tilde{\psi}$ that agrees with the marginal contribution principle in the sense that $\tilde{\psi}_{i}(N, v)=v(N)-v(N \backslash\{i\})$ for every game $\langle N, v\rangle$ and all $i \in N$. Another well-known pseudovalue, called Banzhaf value, corresponds to the uniform weights $p_{s}^{n}=\frac{1}{2^{n-1}}$ for all $0 \leq s \leq n-1$, while the induced pseudovalue is associated with the weights $\tilde{p}_{s}^{n}=\frac{2 \cdot s+2-n}{2^{n-1}}$ for all $0 \leq s \leq n-1$, the smallest weights of which are negative. Because of this observation, we do not want to exclude pseudovalues associated with not necessarily non-negative weights. Throughout the remainder of this section, the induced pseudovalue turns out to be of particular interest in order to provide an appealing explicit and implicit interpretation of the modified reduced game. 
In the second stage we claim two preliminary results each of which is of interest on its own. Firstly, by (3.6), we state that the mapping $F_{\psi}$ induced by an initial pseudovalue $\psi$ may be interpreted as the potential function of the induced pseudovalue $\tilde{\psi}$ (in the sense that $P_{\tilde{\psi}}(N, v):=F_{\psi}^{v}(N)$ for every game $\left.\langle N, v\rangle\right)$. Secondly, by (3.7), in comparing the two solution games associated with the modified reduced game and the initial game respectively, the increase (decrease) to the worth of any coalition turns out to be coalitionally-size-proportional to the increase (decrease) to the payoff of the removed player, taking into account his initial payoff and his payoff according to the induced pseudovalue $\tilde{\psi}$ (with respect to the subgame the player set of which consists of the partnership between the coalition involved and the removed player).

In the third and final stage we claim, by (3.10), that a specifically chosen weighted sum of the latter increases (decreases) to the payoff of the removed player represents the increase (decrease) to the worth of any coalition, in comparing the modified reduced game and the initial game respectively. The recursively computable coefficients used in the relevant weighted sum are identical to those which appear in the explicit determination of the inverse of the bijective mapping $F_{\psi}$ associated with the pseudovalue $\psi$. This mapping turns out to be bijective under very mild conditions imposed upon the underlying collection of weights $\mathcal{P}$ that prescribe the pseudovalue $\psi$.

Theorem 3.3. Let $\psi$ be a pseudovalue on $\mathcal{G}$ of the form (3.1) associated with the collection of weights $\mathcal{P}=\left\{p_{s}^{n}\right\}$. Let $F_{\psi}: \mathcal{G} \rightarrow \mathcal{G}$ be the induced mapping as given by (2.2). Further, let $\tilde{\psi}$ be the induced pseudovalue on $\mathcal{G}$ associated with the induced collection of weights $\tilde{\mathcal{P}}=\left\{\tilde{p}_{s}^{n}\right\}$ as given by (3.4). Then the following holds:

$$
\begin{aligned}
& F_{\psi}^{v}(T)-F_{\psi}^{v}(T \backslash\{i\})=\tilde{\psi}_{i}(T, v) \quad \text { for all }\langle N, v\rangle \in \mathcal{G} \text {, all } T \subseteq N, \text { all } i \in T . \\
& F_{\psi}^{\left(\tilde{v}_{N \backslash\{i\}}^{y_{i}}\right)}(S)-F_{\psi}^{v}(S)=\frac{s}{n-1} \cdot\left[\tilde{\psi}_{i}(S \cup\{i\}, v)-y_{i}\right]
\end{aligned}
$$

for all $\langle N, v\rangle \in \mathcal{G}$, all $i \in N$, all $S \subseteq N \backslash\{i\}$, and all $y_{i} \in \mathbb{R}$ (provided $n \geq 2$ ).

\section{Proof of Theorem 3.3.}

(i) Let $\langle N, v\rangle \in \mathcal{G}, T \subseteq N$ and $i \in T$. By (3.3), player $i$ 's incremental return with respect to the coalition $T$ in the associated solution game $\left\langle N, F_{\psi}^{v}\right\rangle$ is determined as follows:

$$
\begin{aligned}
F_{\psi}^{v}(T)-F_{\psi}^{v}(T \backslash\{i\}) & \stackrel{(3.3)}{=} \\
& \sum_{S \subseteq T} \tilde{p}_{s-1}^{t} \cdot v(S)-\sum_{S \subseteq T \backslash\{i\}} \tilde{p}_{s-1}^{t-1} \cdot v(S) \\
& =\sum_{\substack{S \subseteq T, S \ni i}} \tilde{p}_{s-1}^{t} \cdot v(S)+\sum_{S \subseteq T \backslash\{i\}}\left[\tilde{p}_{s-1}^{t}-\tilde{p}_{s-1}^{t-1}\right] \cdot v(S) \\
& \sum_{S \subseteq T \backslash\{i\}} \tilde{p}_{s}^{t} \cdot v(S \cup\{i\})-\sum_{S \subseteq T \backslash\{i\}} \tilde{p}_{s}^{t} \cdot v(S) \\
& =\sum_{S \subseteq T \backslash\{i\}} \tilde{p}_{s}^{t} \cdot[v(S \cup\{i\})-v(S)] \stackrel{(3.1)}{=} \tilde{\psi}_{i}(T, v)
\end{aligned}
$$

where the third equality is due to the upwards triangle property for $\tilde{\mathcal{P}}$ (see Proposition 3.2(ii)). (ii) Let $\langle N, v\rangle \in \mathcal{G}, i \in N$, and $y_{i} \in \mathbb{R}$ (provided $n \geq 2$ ). ¿From the implicit definition of the 
modified reduced game as given by (2.6), and (3.6) applied to $T=S \cup\{i\}$ respectively, we derive the following:

$$
\begin{aligned}
F_{\psi}^{\left(\tilde{v}_{N \backslash\{i\}}^{y_{i}}\right)}(S)-F_{\psi}^{v}(S) & \stackrel{\stackrel{(2.6)}{=}}{=} \frac{s}{n-1} \cdot\left[F_{\psi}^{v}(S \cup\{i\})-F_{\psi}^{v}(S)-y_{i}\right] \\
& \stackrel{(3.6)}{=} \frac{s}{n-1} \cdot\left[\tilde{\psi}_{i}(S \cup\{i\}, v)-y_{i}\right] \quad \text { for all } S \subseteq N \backslash\{i\} .
\end{aligned}
$$

Theorem 3.4. Let $\psi$ be a pseudovalue on $\mathcal{G}$ of the form (3.1) associated with the collection of weights $\mathcal{P}=\left\{p_{s}^{n}\right\}$ satisfying $p_{n-1}^{n}>0$ for all $n \geq 1$. Let $F_{\psi}: \mathcal{G} \rightarrow \mathcal{G}$ be the induced mapping as given by (2.2). Further, let $\tilde{\mathcal{P}}=\left\{\tilde{p}_{s}^{n}\right\}$ be the induced collection of weights as given by (3.4). For every $t \geq 1$, let the induced collection of constants $\left\{q_{k}^{t}(s) \in \mathbb{R} \mid k \in\right.$ $\{1,2, \ldots, t\}, s \in\{1,2, \ldots, t+1-k\}\}$ be defined recursively by

$$
\begin{aligned}
q_{1}^{t}(s) & :=\tilde{p}_{s-1}^{t} \quad \text { for all } 1 \leq s \leq t \quad \text { and } \\
q_{k+1}^{t}(s) & :=\left(\begin{array}{c}
t-s \\
k
\end{array}\right) \cdot \frac{\tilde{p}_{s-1}^{t-k}}{\tilde{p}_{t-1-k}^{t-k}} \cdot q_{k}^{t}(t-k)-q_{k}^{t}(s) \quad \text { for all } 1 \leq k \leq t-1, \text { all } 1 \leq s \leq t-k .
\end{aligned}
$$

Then the following holds:

(i) Given that $F_{\psi}^{v}(T)=\sum_{S \subseteq T} \tilde{p}_{s-1}^{t} \cdot v(S)$ for every game $\langle N, v\rangle$ and all $T \subseteq N$ (see (3.3)), the data $v(T), T \subseteq N$, of any game $\langle N, v\rangle$ can be re-discovered as follows:

$$
\tilde{p}_{t-1}^{t} \cdot v(T)=\sum_{\substack{S \subseteq T \\ S \neq \emptyset}}(-1)^{t-s} \cdot \frac{q_{t-s}^{t}(s)}{\tilde{p}_{s-1}^{s}} \cdot F_{\psi}^{v}(S) \quad \text { for all } T \subseteq N, \quad \text { where } q_{0}^{t}(t):=\tilde{p}_{t-1}^{t}
$$

(ii) Let $\tilde{\psi}$ be the induced pseudovalue on $\mathcal{G}$ associated with $\tilde{\mathcal{P}}=\left\{\tilde{p}_{s}^{n}\right\}$. Then it holds

$$
\tilde{p}_{t-1}^{t} \cdot\left[\tilde{v}_{N \backslash\{i\}}^{y_{i}}(T)-v(T)\right]=\sum_{\substack{S \subseteq T, S \neq \emptyset}}(-1)^{t-s} \cdot \frac{q_{t-s}^{t}(s)}{\tilde{p}_{s-1}^{s}} \cdot \frac{s}{n-1} \cdot\left[\tilde{\psi}_{i}(S \cup\{i\}, v)-y_{i}\right]
$$

for all $\langle N, v\rangle \in \mathcal{G}$, all $i \in N$, all $T \subseteq N \backslash\{i\}, T \neq \emptyset$, and all $y_{i} \in \mathbb{R}$ (provided $n \geq 2$ ).

(iii) For the special case $p_{s}^{n}=\frac{1}{n \cdot\left(\begin{array}{c}n-1 \\ s\end{array}\right)}$, then (3.10) reduces to Sobolev's reduced game (2.4).

The rather technical proof of Theorem 3.4 will be postponed till the appendix.

Remark 3.5. To conclude with, we specify the explicit determination for the worth of oneand two-person coalitions in the $(n-1)$-person modified reduced game (2.6), without regard to the number $n$ of players in the initial game.

Let $\psi$ be a pseudovalue on $\mathcal{G}$ of the form (3.1) associated with the collection of weights $\mathcal{P}=\left\{p_{s}^{n}\right\}$ satisfying $p_{n-1}^{n}>0$ for all $n \geq 1$. Let $\tilde{\psi}$ be the induced pseudovalue on $\mathcal{G}$ associated with the induced collection of weights $\tilde{\mathcal{P}}=\left\{\tilde{p}_{s}^{n}\right\}$ as given by (3.4). 
Consider an arbitrary $n$-person game $\langle N, v\rangle$ and let $i \in N, y_{i} \in \mathbb{R}$. By applying (3.10) to oneand two-person coalitions $T=\{j\}$ and $T=\{j, k\}$ respectively, and (3.1) to the pseudovalue $\tilde{\psi}$, we obtain that the worth of one- and two-person coalitions in the $(n-1)$-person modified reduced game $\left\langle N \backslash\{i\}, \tilde{v}_{N \backslash\{i\}}^{y_{i}}\right\rangle$ of the form (2.6) is determined as follows (recall that, by (3.8), $\left.q_{1}^{2}(1)=\tilde{p}_{0}^{2}\right)$ :

$$
\begin{aligned}
\tilde{v}_{N \backslash\{i\}}^{y_{i}}(\{j\}) & =v(\{j\})+\frac{1}{(n-1) \cdot \tilde{p}_{0}^{1}} \cdot\left[\tilde{p}_{1}^{2} \cdot[v(\{i, j\})-v(\{j\})]+\tilde{p}_{0}^{2} \cdot v(\{i\})-y_{i}\right] \\
\tilde{v}_{N \backslash\{i\}}^{y_{i}}(\{j, k\}) & =v(\{j, k\})+\frac{2 \cdot \tilde{p}_{2}^{3}}{(n-1) \cdot \tilde{p}_{1}^{2}} \cdot[v(\{i, j, k\})-v(\{j, k\})] \\
& +\frac{\left[2 \cdot \tilde{p}_{1}^{3} \cdot \tilde{p}_{0}^{1}-\tilde{p}_{1}^{2} \cdot \tilde{p}_{0}^{2}\right]}{(n-1) \cdot \tilde{p}_{1}^{2} \cdot \tilde{p}_{0}^{1}} \cdot[v(\{i, j\})-v(\{j\})+v(\{i, k\})-v(\{k\})] \\
& +\frac{2 \cdot\left[\tilde{p}_{0}^{3} \cdot \tilde{p}_{0}^{1}-\tilde{p}_{0}^{2} \cdot \tilde{p}_{0}^{2}\right]}{(n-1) \cdot \tilde{p}_{1}^{2} \cdot \tilde{p}_{0}^{1}} \cdot v(\{i\})+\frac{2 \cdot\left[\tilde{p}_{0}^{2}-\tilde{p}_{0}^{1}\right]}{(n-1) \cdot \tilde{p}_{1}^{2} \cdot \tilde{p}_{0}^{1}} \cdot y_{i}
\end{aligned}
$$

In the framework of three-person games, we obtained a complete description of the two-person modified reduced game and by tedious, but straightforward calculations, one may verify that the consistency property $\psi_{j}\left(N \backslash\{i\}, \tilde{v}_{N \backslash\{i\}}^{\psi_{i}(N, v)}\right)=\psi_{j}(N, v)$ holds true for the pseudovalue $\psi$ with respect to three-person games. One useful tool concerns the upwards triangle property for $\tilde{\mathcal{P}}$.

Remark 3.6. The relationship (3.6) is also useful to provide, in the framework of pseudovalues, an alternative proof of the fundamental equivalence theorem between any pseudovalue $\psi$ and the Shapley value, that is $\psi(N, v)=S h\left(N, F_{\psi}^{v}\right)$ for every game $\langle N, v\rangle$. Let us outline this alternative proof that differs from Calvo's proof (cf. [1]) and Sánchez' proof (cf. [11]) of the equivalence theorem applied to solutions that admit a potential.

Let $\langle N, v\rangle \in \mathcal{G}$. Recall that, by straightforward combinatorial computations, the solution game $\left\langle N, F_{\psi}^{v}\right\rangle$ is determined by (3.3) and in turn, the incremental returns of any player in the solution game are determined by (3.6), i.e.,

$$
F_{\psi}^{v}(T \cup\{i\})-F_{\psi}^{v}(T) \stackrel{(3.6)}{=} \tilde{\psi}_{i}(T \cup\{i\}, v) \stackrel{(3.1)}{=} \sum_{R \subseteq T} \tilde{p}_{r}^{t+1} \cdot[v(R \cup\{i\})-v(R)]
$$

for all $i \in N$ and all $T \subseteq N \backslash\{i\}$. From this and some additional combinatorial computations, we deduce that, for all $i \in N$, the following chain of equalities holds:

$$
\begin{aligned}
S h_{i}\left(N, F_{\psi}^{v}\right) & \stackrel{(2.3)}{=} \sum_{T \subseteq N \backslash\{i\}} \frac{1}{n \cdot\left(\begin{array}{c}
n-1 \\
t
\end{array}\right)} \cdot\left[F_{\psi}^{v}(T \cup\{i\})-F_{\psi}^{v}(T)\right] \\
& =\sum_{T \subseteq N \backslash\{i\}} \frac{1}{n \cdot\left(\begin{array}{c}
n-1 \\
t
\end{array}\right)} \cdot \sum_{R \subseteq T} \tilde{p}_{r}^{t+1} \cdot[v(R \cup\{i\})-v(R)] \\
& =\sum_{S \subseteq N \backslash\{i\}}\left[\sum_{t=s}^{n-1}\left(\begin{array}{c}
n-1-s \\
t-s
\end{array}\right) \cdot \frac{1}{n \cdot\left(\begin{array}{c}
n-1 \\
t
\end{array}\right)} \cdot \tilde{p}_{s}^{t+1}\right] \cdot[v(S \cup\{i\})-v(S)] \\
& =\sum_{S \subseteq N \backslash\{i\}} p_{s}^{n} \cdot[v(S \cup\{i\})-v(S)] \stackrel{(3.1)}{=} \psi_{i}(N, v)
\end{aligned}
$$


For the sake of the last equality but one, we need to establish the following claim:

$\sum_{t=s}^{n-1}\left(\begin{array}{c}n-1-s \\ t-s\end{array}\right) \cdot \frac{\tilde{p}_{s}^{t+1}}{n \cdot\left(\begin{array}{c}n-1 \\ t\end{array}\right)}=p_{s}^{n} \quad$ or equivalently, $\quad \sum_{t=s}^{n-1}\left(\begin{array}{c}t \\ s\end{array}\right) \cdot \tilde{p}_{s}^{t+1}=(n-s) \cdot\left(\begin{array}{l}n \\ s\end{array}\right) \cdot p_{s}^{n}$

for all $0 \leq s \leq n-1$. The proof of the claim (3.11) proceeds by induction on the size $n$, $n \geq s+1$, where $s \geq 0$ is fixed. Recall (3.4) and the upwards triangle property of $\mathcal{P}=\left\{p_{s}^{n}\right\}$. The inductive proof of (3.11) is left to the reader.

\section{References}

[1] Calvo, E., and J.C. Santos, (1997), Potentials in cooperative TU-games. Mathematical Social Sciences 34, 175-190.

[2] Dragan, I., (1996), New mathematical properties of the Banzhaf value. European Journal of Operational Research 95, 451-463.

[3] Driessen, T.S.H., (1988), Cooperative Games,Solutions, and Applications. Kluwer Academic Publishers, Dordrecht, The Netherlands.

[4] Driessen, T.S.H., (1991), A survey of consistency properties in cooperative game theory. SIAM Review 33, 43-59.

[5] Dubey, P., Neyman, A., and R.J. Weber, (1981), Value theory without efficiency. Mathematics of Operations Research 6, 122-128.

[6] Hart, S., and A. Mas-Colell, (1989), Potential, value, and consistency. Econometrica 57, 589-614. Also in [10], 127-137.

[7] Maschler, M., (1992), The bargaining set, kernel, and nucleolus. In: Handbook of Game Theory with Economic Applications, Volume 1 (Eds. R.J. Aumann and S. Hart). Handbooks in Economics 11, Elsevier Science Publishers, Amsterdam, The Netherlands. Chapter 18, 591-667. See also Chapter 13 by Peleg, B., Axiomatizations of the Core, 397-412.

[8] Myerson, R., (1980), Conference structures and fair allocation rules. International Journal of Game Theory 9, 169-182.

[9] Ortmann, K.M., (1998), Conservation of energy in value theory. Mathematical Methods of Operations Research 47, 423-450.

[10] Roth, A.E. (editor), (1988), The Shapley value: Essays in honor of Lloyd S. Shapley. Cambridge University Press, Cambridge, U.S.A.

[11] Sánchez S., F., (1997), Balanced contributions in the solution of cooperative games. Games and Economic Behavior 20, 161-168.

[12] Shapley, L.S., (1953), A value for n-person games. Annals of Mathematics Study 28, 307-317 (Princeton University Press). Also in [10], 31-40.

[13] Sobolev, A.I., (1973), The functional equations that give the payoffs of the players in an n-person game (in Russian). In: Advances in Game Theory (Ed. E. Vilkas). Izdat. "Mintis", Vilnius, 151-153. 


\section{APPENDIX: Two technical proofs}

\section{Proof of Proposition 3.2.}

(i) Let $\langle N, v\rangle \in \mathcal{G}$ and $T \subseteq N, T \neq \emptyset$. By assumption of a pseudovalue $\psi$ of the form (3.1) applied to the subgame $\langle T, v\rangle$, and by some straightforward combinatorial computations, we obtain

$$
\begin{aligned}
F_{\psi}^{v}(T) \stackrel{(2.2)}{=} \sum_{i \in T} \psi_{i}(T, v) & \stackrel{(3.1)}{=} \sum_{i \in T} \sum_{S \subseteq T \backslash\{i\}} p_{s}^{t} \cdot[v(S \cup\{i\})-v(S)] \\
& =\sum_{S \subseteq T}\left[s \cdot p_{s-1}^{t}-(t-s) \cdot p_{s}^{t}\right] \cdot v(S)
\end{aligned}
$$

(ii) Let $n \geq 1$. By (3.4) and the upwards triangle property (3.2) for $\mathcal{P}=\left\{p_{s}^{n}\right\}$, it holds

$$
\begin{aligned}
& \tilde{p}_{s}^{n+1}+\tilde{p}_{s+1}^{n+1} \stackrel{(3.4)}{=}\left[(s+1) \cdot p_{s}^{n+1}-(n-s) \cdot p_{s+1}^{n+1}\right]+\left[(s+2) \cdot p_{s+1}^{n+1}-(n-1-s) \cdot p_{s+2}^{n+1}\right] \\
& =(s+1) \cdot\left[p_{s}^{n+1}+p_{s+1}^{n+1}\right]-(n-1-s) \cdot\left[p_{s+1}^{n+1}+p_{s+2}^{n+1}\right] \\
& \stackrel{(3.2)}{=}(s+1) \cdot p_{s}^{n}-(n-1-s) \cdot p_{s+1}^{n} \stackrel{(3.4)}{=} \tilde{p}_{s}^{n} \quad \text { for all } 0 \leq s \leq n-1 .
\end{aligned}
$$

(iii) Fix $n \geq 1$. The proof of (3.5) proceeds by backwards induction on the size $s, 0 \leq s \leq n-1$. For $s=n-1$, (3.5) holds because of $\tilde{p}_{n-1}^{n}=n \cdot p_{n-1}^{n}$. For $1 \leq s \leq n-1$, we deduce from (3.4) and the induction hypothesis applied to $s$ that it holds

$$
\begin{aligned}
p_{s-1}^{n} & \stackrel{(3.4)}{=} \quad \frac{\tilde{p}_{s-1}^{n}}{s}+\frac{n-s}{s} \cdot p_{s}^{n} \\
& =\frac{\tilde{p}_{s-1}^{n}}{s}+\frac{n-s}{s} \cdot \frac{1}{n \cdot\left(\begin{array}{c}
n-1 \\
s
\end{array}\right)} \cdot \sum_{t=s}^{n-1}\left(\begin{array}{c}
n \\
t+1
\end{array}\right) \cdot \tilde{p}_{t}^{n} \\
& =\frac{1}{n \cdot\left(\begin{array}{c}
n-1 \\
s-1
\end{array}\right)} \cdot \sum_{t=s-1}^{n-1}\left(\begin{array}{c}
n \\
t+1
\end{array}\right) \cdot \tilde{p}_{t}^{n}
\end{aligned}
$$

(iv) For every $n \geq 1$ and $0 \leq s \leq n-1$, write $\beta_{s}^{n}:=\frac{1}{n \cdot\left(\begin{array}{c}n-1 \\ s\end{array}\right)}$. Let $n \geq 1$ and $0 \leq s \leq n-1$.

On the one hand, we deduce from the assumption (3.5) that it holds

$$
\begin{aligned}
p_{s}^{n+1}+p_{s+1}^{n+1} & \stackrel{(3.5)}{=} \beta_{s}^{n+1} \cdot \sum_{t=s}^{n}\left(\begin{array}{c}
n+1 \\
t+1
\end{array}\right) \cdot \tilde{p}_{t}^{n+1}+\beta_{s+1}^{n+1} \cdot \sum_{t=s+1}^{n}\left(\begin{array}{c}
n+1 \\
t+1
\end{array}\right) \cdot \tilde{p}_{t}^{n+1} \\
& =\beta_{s}^{n+1} \cdot\left(\begin{array}{c}
n+1 \\
s+1
\end{array}\right) \cdot \tilde{p}_{s}^{n+1}+\left[\beta_{s}^{n+1}+\beta_{s+1}^{n+1}\right] \cdot \sum_{t=s+1}^{n}\left(\begin{array}{c}
n+1 \\
t+1
\end{array}\right) \cdot \tilde{p}_{t}^{n+1} \\
& =\frac{\tilde{p}_{s}^{n+1}}{s+1}+\beta_{s}^{n} \cdot \sum_{t=s+1}^{n}\left(\begin{array}{c}
n+1 \\
t+1
\end{array}\right) \cdot \tilde{p}_{t}^{n+1}
\end{aligned}
$$


On the other hand, we deduce from the upwards triangle property for $\tilde{\mathcal{P}}=\left\{\tilde{p}_{s}^{n}\right\}$ that it holds

$$
\begin{aligned}
p_{s}^{n} \stackrel{\stackrel{(3.5)}{=}}{=} \beta_{s}^{n} \cdot \sum_{t=s}^{n-1}\left(\begin{array}{c}
n \\
t+1
\end{array}\right) \cdot \tilde{p}_{t}^{n} \\
=\beta_{s}^{n} \cdot \sum_{t=s}^{n-1}\left(\begin{array}{c}
n \\
t+1
\end{array}\right) \cdot\left[\tilde{p}_{t}^{n+1}+\tilde{p}_{t+1}^{n+1}\right] \\
=\beta_{s}^{n} \cdot\left(\begin{array}{c}
n \\
s+1
\end{array}\right) \cdot \tilde{p}_{s}^{n+1}+\beta_{s}^{n} \cdot \sum_{t=s+1}^{n-1}\left[\left(\begin{array}{c}
n \\
t+1
\end{array}\right)+\left(\begin{array}{c}
n \\
t
\end{array}\right)\right] \cdot \tilde{p}_{t}^{n+1}+\beta_{s}^{n} \cdot \tilde{p}_{n}^{n+1} \\
=\frac{\tilde{p}_{s}^{n+1}}{s+1}+\beta_{s}^{n} \cdot \sum_{t=s+1}^{n}\left(\begin{array}{c}
n+1 \\
t+1
\end{array}\right) \cdot \tilde{p}_{t}^{n+1}
\end{aligned}
$$

Since both computational methods yield the very same outcome, we conclude that $p_{s}^{n+1}+$ $p_{s+1}^{n+1}=p_{s}^{n}$. Finally, the statement in part (v) is a direct consequence of (3.4).

Proof of Theorem 3.4. Let $\langle N, v\rangle \in \mathcal{G}$.

(i) For every $T \subseteq N, T \neq \emptyset$, it suffices to prove the next equality:

$$
\sum_{\substack{S \subsetneq T, S \neq \emptyset}} \tilde{p}_{s-1}^{t} \cdot v(S)=\sum_{\substack{S \subsetneq T, S \neq \emptyset}}(-1)^{t+1-s} \cdot \frac{q_{t-s}^{t}(s)}{\tilde{p}_{s-1}^{s}} \cdot F_{\psi}^{v}(S)
$$

Fix $T \subseteq N$ and $S \varsubsetneqq T, S \neq \emptyset$, as well. We aim to determine the coefficient of the term $v(S)$ in the sum given by the right hand of (4.1). The term $v(S)$ occurs in any expression $F_{\psi}^{v}(R)$ as long as $S \subseteq R$, provided that $R \varsubsetneqq T$. Thus, we need only to consider those coalitions $R$ satisfying $S \subseteq R \varsubsetneqq T$ and each such coalition $R$, say of size $r, s \leq r \leq t-1$, induces the term

$$
(-1)^{t+1-r} \cdot \frac{q_{t-r}^{t}(r)}{\tilde{p}_{r-1}^{r}} \cdot \tilde{p}_{s-1}^{r} \cdot v(S)
$$

Notice that, for any size $r, s \leq r \leq t-1$, there exists $\left(\begin{array}{c}t-s \\ t-r\end{array}\right)$ coalitions $R$ of size $r$ satisfying $S \subseteq R \varsubsetneqq T$. Hence, for every fixed $S \varsubsetneqq T, S \neq \emptyset$, the coefficient of the term $v(S)$ in the sum given by the right hand of (4.1) is determined by the next sum:

$$
\sum_{r=s}^{t-1}\left(\begin{array}{c}
t-s \\
t-r
\end{array}\right) \cdot(-1)^{t+1-r} \cdot q_{t-r}^{t}(r) \cdot \frac{\tilde{p}_{s-1}^{r}}{\tilde{p}_{r-1}^{r}}
$$

By construction based on (3.8), for all $1 \leq s \leq t-1$, it holds

$$
\begin{aligned}
& \sum_{r=s}^{t-1}\left(\begin{array}{c}
t-s \\
t-r
\end{array}\right) \cdot(-1)^{t+1-r} \cdot q_{t-r}^{t}(r) \cdot \frac{\tilde{p}_{s-1}^{r}}{\tilde{p}_{r-1}^{r}}=\sum_{k=1}^{t-s}\left(\begin{array}{c}
t-s \\
k
\end{array}\right) \cdot(-1)^{k+1} \cdot q_{k}^{t}(t-k) \cdot \frac{\tilde{p}_{s-1}^{t-k}}{\tilde{p}_{t-1-k}^{t-k}} \\
& \stackrel{(3.8)}{=} \sum_{k=1}^{t-s}(-1)^{k+1} \cdot\left[q_{k+1}^{t}(s)+q_{k}^{t}(s)\right] \\
& =q_{1}^{t}(s)+(-1)^{t+1-s} \cdot q_{t+1-s}^{t}(s) \\
& \stackrel{(3.8)}{=} \tilde{p}_{s-1}^{t}+0=\tilde{p}_{s-1}^{t} \quad \text { This proves }(4.1) \text {. }
\end{aligned}
$$


(ii) (3.10) is a direct consequence of both (3.7) and (3.9) applied to the initial game and the reduced game as well.

(iii) By (3.4), $p_{s}^{n}=\frac{1}{n \cdot\left(\begin{array}{c}n-1 \\ s\end{array}\right)}$ implies $\tilde{p}_{n-1}^{n}=1$ and $\tilde{p}_{s}^{n}=0$ for all $n \geq 1$, all $0 \leq s \leq n-2$.

By (3.8), $q_{1}^{t}(s)=0$ whenever $1 \leq s \leq t-1$ and thus, $q_{k}^{t}(s)=0$ whenever $k \geq 2$. Therefore, (3.10) reduces to the next equality:

$$
\begin{aligned}
\tilde{v}_{N \backslash\{i\}}^{y_{i}}(T)-v(T) & \stackrel{(3.10)}{=} \frac{t}{n-1} \cdot\left[\tilde{\psi}_{i}(T \cup\{i\}, v)-y_{i}\right] \\
& \stackrel{(3.1)}{=} \frac{t}{n-1} \cdot\left[v(T \cup\{i\})-v(T)-y_{i}\right]
\end{aligned}
$$

Obviously, the relevant equality agrees with Sobolev's reduced game (2.4). 